\title{
Estudo Demográfico do Câncer de Canal Anal e Ânus no Estado de Sergipe
}

\author{
Cancer of Canal Anal and Anus. A Demografic \\ Study in Sergipe - Brasil
}

\author{
JUVENALDAROCHATORRES NETO ${ }^{1}$, ANACAROLINALISBÔAPRUDENTE² ${ }^{2}$ RONMELLISBOADOS SANTOS \\ ${ }^{1}$ Professor, Doutor e Chefe do Serviço de Colo-proctologia; ${ }^{2}$ Médica residente em colo-proctologia. Hospital \\ Universitário 3 Acadêmico de medicina da Universidade Federal de Sergipe - BRASIL
}

TORRES NETO JR; PRUDENTE ACL; SANTOS, RL. Estudo demográfico do câncer de canal anal e ânus no estado de Sergipe. Rev bras Coloproct, 2007;27(2): 190-195.

RESUMO: A carência de estudos demográficos específicos sobre o câncer de canal anal e ânus no Brasil e uma melhor compreensão do comportamento da patologia no estado de Sergipe motivaram a realização de tal estudo. Em análise retrospectiva (1993 - 2005), foram avaliadas as principais características demográficas do câncer anal em Sergipe. Foram estudados 91 pacientes, sendo 70 (76,9\%) do gênero feminino e $21(23,0 \%)$ do gênero masculino. A média de casos novos ao ano no período estudado foi de 0,39 por 100.000 pessoas, com desvio padrão de $\pm 0,16$. O carcinoma de células escamosas (CEC) foi responsável por $68 \%$ dos casos de câncer anal. No presente estudo, constatou-se o mau preenchimento dos prontuários médicos e a necessidade de se criar um protocolo para o atendimento e acompanhamento dos pacientes com câncer de canal anal e ânus.

Descritores: Câncer, Câncer anal, Estudo demográfico.

\section{INTRODUÇÃO}

Os tumores malignos do ânus e canal anal são entidades raras, não ultrapassando a taxa de $2 \%$ de todos os tumores do intestino grosso ${ }^{1}$ e cerca de $3 \%$ a $3,5 \%$ dos tumores anorretais ${ }^{2}$. A incidência de neoplasias de cólon, reto e ânus para 2006, no Brasil, foi estimada para cerca de 11.390 novos casos no gênero masculino, e 13.970 novos casos no gênero feminino $^{3}$, porém não se dispõe de dados específicos do câncer anal no país.

Um estudo realizado na Califórnia registrou 4.841 pacientes com diagnóstico de câncer de canal anal no período de 1973 a 1998. Esse trabalho constata uma maior incidência no gênero feminino, havendo um crescimento da incidência, no período estudado, em ambos os gêneros ${ }^{4}$. Outra pesquisa realizada na Califórnia também encontrou predominância dos casos no gênero feminino, mas em idades inferiores a $40-44$ anos a incidência era maior entre os homens ${ }^{5}$.

Em um estudo brasileiro, Nakajima et al., analisaram 58 pacientes com câncer anal. A média de idade foi de 61 anos, sendo a faixa etária mais acometida entre 60 e 64 anos. Quanto ao gênero, 70,7\% eram feminino e 29,3\%, masculino, sendo a proporção de 2,4 mulheres para cada homem 6 .

No ânus ou margem anal podem se desenvolver carcinoma espinocelular (CEC), carcinoma de células basais, doença de Bowen, carcinoma verrucoso ou tumor de Buschke-Löwsnstein, sarcoma de Kaposi e doença de Paget perianal. Os tumores malignos do canal anal são de quatro naturezas histológicas: CEC, melanoma maligno, adenocarcinoma e sarcomas ${ }^{1}$. O CEC representa a maioria dos tumores com cerca de $80 \%$ dos $\operatorname{casos}^{2,7}$.

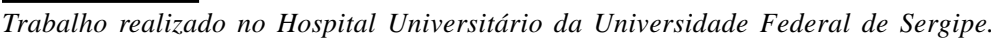

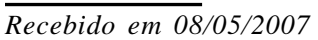

Aceito para publicação em 29/05/2007 
O tratamento combinado com radioquimioterapia passou a ser o tratamento de escolha para o câncer anal desde a década de 70 após o trabalho de Nigro, reservando a amputação abdomino-perineal para os casos de doença persistente ou recorrência local $^{8}$.

A importância dessa pesquisa reside na escassez de dados demográficos específicos sobre câncer de canal anal e ânus, já que a literatura aborda esse assunto em conjunto com os tumores do intestino grosso. O objetivo do trabalho é fazer um levantamento de dados sobre os tumores malignos de canal anal e ânus no estado de Sergipe entre 1993 e 2005, analisando variáveis como incidência, gênero, idade, estado civil, tipo histológico e grau de diferenciação.

\section{PACIENTES E MÉTODOS}

Foi realizado um estudo retrospectivo de todos os pacientes com diagnóstico de câncer de canal anal e ânus no estado de Sergipe em um período de 13 anos $(1993-2005)$.

Os dados dos pacientes foram coletados em laudos de todos os laboratórios de anatomia patológica de Aracaju, cidade que concentra a totalidade dos laboratórios de anátomo-patologia do estado de Sergipe. Foram preenchidos os protocolos previamente elaborados (anexo III), considerando, principalmente variáveis como: idade, gênero, tipo histológico e grau de diferenciação.

A taxa de incidência anual foi calculada dividindo-se o número de casos novos (numerador) surgido no ano pela população estimada (denominador) no ano considerado. A fonte para se obter a população estimada foi o Instituto Brasileiro de Geografia e Estatística- IBGE $^{9}$. A taxa de incidência anual também foi ajustada para o gênero, utilizando-se nesse caso o número de casos novos anuais e a população estimada por gênero. A incidência foi relatada como o número de casos novos de câncer de canal anal e ânus por 100.000 pessoas.

A análise estatística e os gráficos utilizados no estudo foram desenvolvidos em planilhas do Microsoft Excel $^{\circledR} 2003$.

\section{RESULTADOS}

Em um período de 13 anos, compreendido entre janeiro de 1993 e dezembro de 2005, foram diag- nosticados 91 casos de câncer de canal anal e ânus confirmados pelos exames de anátomo-patologia.

A idade dos pacientes variou de 24 a 91 anos, apresentando uma média de 63,8 anos e desvio padrão de $\pm 5,4$. A sétima e a oitava décadas de vida foram as mais acometidas com um total de 47,5\% (39) dos casos. A média de idade dos pacientes masculinos com câncer anal foi 59,4 anos (26-91), tendo desvio padrão de $\pm 13,77$. Entre as mulheres, a média de idade foi de 66 anos $(24-88)$ e desvio padrão de $\pm 8,0$.

Houve um predomínio no gênero feminino com $70(76,9 \%)$ casos versus $21(23,0 \%)$ no gênero masculino. Esse predomínio diminui nas faixas etárias inferiores a 50 anos, com $15(16,4 \%)$ mulheres para 9 $(9,9 \%)$ homens acometidos por câncer de canal anal e ânus.

Conseguiu-se levantar o estado civil de 44 (48\%) pacientes com câncer anal, dos quais, 24 (26,6\%) eram casados, $10(11 \%)$ viúvos, $9(9,8 \%)$ solteiros e $1(1,1 \%)$ divorciado. Se considerarmos apenas esses 44 pacientes, teríamos uma percentagem de casados de $54,5 \%, 22,7 \%$ de viúvos, $20,4 \%$ de solteiros e $2,2 \%$ de divorciados.

A taxa de incidência variou entre 0,18 e 0,83 por 100.000 pessoas com uma média de 0,39 (desvio padrão de $\pm 0,16$ ) casos novos por ano nos 13 anos (1993 - 2005) considerados no estudo. Em todos os anos estudados, a taxa de incidência foi maior entre as mulheres, variando entre 0,36 e 0,91 por 100.000 pes-

Tabela 1 - Número de casos de câncer anal por ano. ANEXO I

\begin{tabular}{rr}
\hline Ano & Casos \\
\hline 1993 & 4 \\
1994 & 6 \\
1995 & 5 \\
1996 & 3 \\
1997 & 8 \\
1998 & 14 \\
1999 & 10 \\
2000 & 5 \\
2001 & 7 \\
2002 & 6 \\
2003 & 10 \\
2004 & 8 \\
2005 & 5 \\
Total & 91 \\
\hline
\end{tabular}




\section{ANEXO II}

\section{$\longrightarrow$ Incidência por 100.000 pessoas}

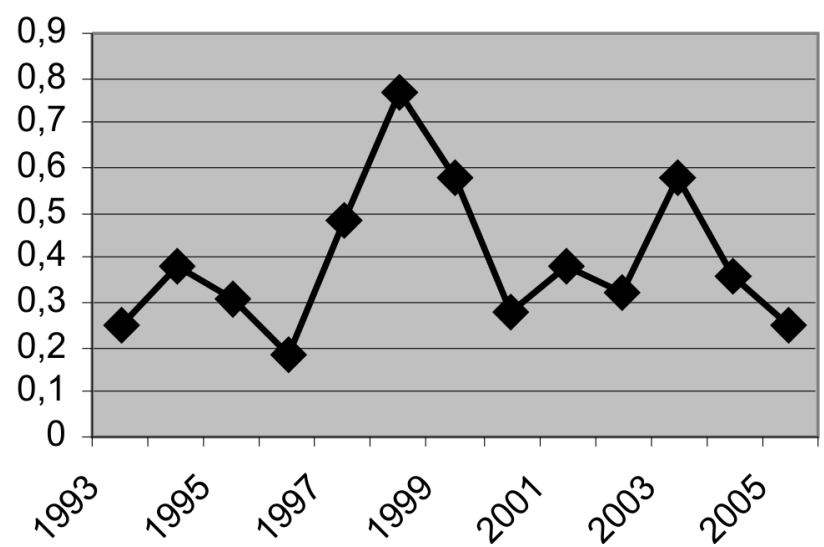

Figura 1 - Taxa de incidência anual do câncer de canal anal e ânus.

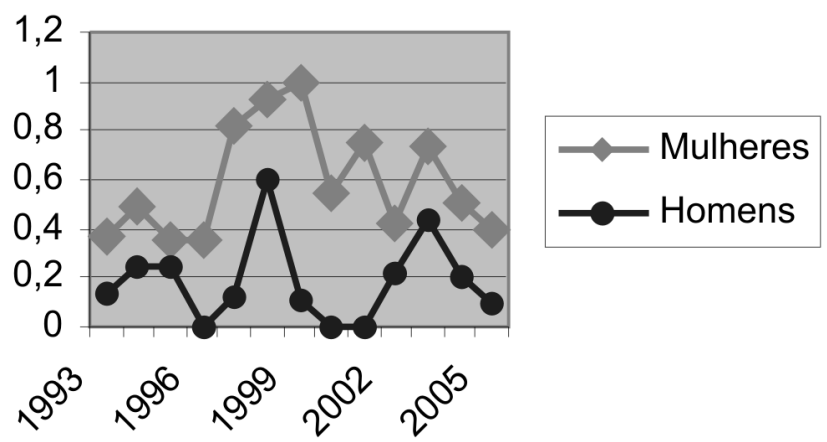

Figura 2 - Taxa de incidência anual de câncer de canal anal e ânus por gênero.

soas (desvio padrão de $\pm 0,21$ ). Entre os homens a maior taxa de incidência foi 0,72 por 100.000 pessoas. Em 3 dos 13 anos estudados não foram relatados casos de câncer anal entre os homens.

$\mathrm{Na}$ amostra estudada, o tipo histológico mais comum foi o carcinoma epidermóide, representando $68,1 \%$ (62) dos tumores. Os outros tipos histológicos em ordem decrescente foram: o cloacogênico 13\% (12), adenocarcinoma com $10,2 \%$ (8), doença de Bowen com 6,6\% (6) e o melanoma com 3,3\% (3). Excluindo-se os casos de adenocarcinoma, teríamos 83 casos de câncer anal, sendo $75 \%$ de carcinoma epidermóide, cloacogênico com $14,4 \%$, doença de Bowen com $9,6 \%$ e o melanoma com $3,6 \%$.

Em relação ao grau de diferenciação, houve $22(24,4 \%)$ bem diferenciados, $31(34,4 \%)$ casos de moderada diferenciação, $9(10 \%)$ pouco diferenciados e $28(31,1 \%)$ casos sem registro desse dado.

\section{DISCUSSÃO}

O câncer de canal anal é uma neoplasia rara, contribuindo com cerca de $1 \%$ a $2 \%$ dos cânceres do intestino grosso ${ }^{10}$ e 3 a $3,5 \%$ dos tumores anorretais ${ }^{2}$. A incidência do câncer anal epidermóide aumentou consideravelmente nas últimas décadas, mais notavelmente entre as mulheres, homens solteiros e pessoas que vivem em áreas urbanas ${ }^{2}$.

A presente pesquisa representa uma casuística importante, onde foram catalogados 91 casos de câncer de ânus diagnosticados em 13 anos no estado de Sergipe (Tabela 1 - anexo I). Essa amostra corresponde a quase totalidade de casos, visto que foi acessado o banco de dados de todos os laboratórios de anátomo-patologia.

Houve uma grande dificuldade em levantar dados na literatura sobre as características demográficas do câncer anal. Em geral, os trabalhos sobre esse assunto têm casuísticas pequenas. No Brasil, Guimarães et al. analisaram retrospectivamente 11 casos de câncer anal entre 1995 e $1999^{11}$, Nakajima et al., em 2001, em estudo caso-controle analisou 58 casos de câncer anal ${ }^{6}$ e Larageira et al. relataram 13 pacientes entre 1998 e $2002^{12}$. Como já foi relatado, o câncer anal é bastante raro. Um estudo realizado na Califórnia obteve o registro de 4.841 pacientes com diagnóstico de câncer de canal anal no período de 1973 a $1998^{4}$.

Em nosso trabalho, a média de casos novos surgidos por ano foi de 0,39 por 100.000 pessoas, variando de 0,18 a 0,91 (Figura 1 - anexo II). Houve uma oscilação na incidência e não um crescimento significativo no período estudado embora, alguns trabalhos, venham relatando um aumento na incidência do câncer anal nas últimas décadas ${ }^{9,13}$. Maggard et al., analisando 4.841 pacientes entre 1973 e 1998, relataram um aumento significativo da incidência de câncer anal, passando de 0,35 para 0,98 por 100.000 pessoas.

A incidência de neoplasias de cólon, reto e ânus para 2006, no Brasil, foi estimada para cerca de 11.390 novos casos no gênero masculino, e 13.970 novos casos no gênero feminino ${ }^{3}$, porém não se dispõe de dados específicos do câncer anal no país ${ }^{3}$.

Em todos os anos estudados na pesquisa, a incidência foi maior entre as mulheres, porém em idades inferiores a 50 anos havia um maior equilíbrio na taxa de incidência entre os dois gêneros (Figura 2 - anexo II). Esses dados estão de acordo com a maioria das séries analisadas ${ }^{4,10}$. 
Juvenal da Rocha Torres Neto e Cols.

ANEXO III

PROTOCOLO DE PESQUISA

\section{Avaliação Epidemiológica do Câncer de Ânus e Canal anal em Sergipe}

Nome: Registro:

Idade:

D. N.:

Gênero:

Est. Civil: Cor: Profissão:

Vida Sodômica: Ocupação:

Endereço:

Naturalidade: Procedência:

Data do Exame:

\section{Quadro Clínico:}

- Dor ano-retal

- Sangramento retal

- Tenesmo

- $\quad$ Prurido

\section{Anátomo-patológico:}

- $\quad$ Tipo histológico:

- Câncer de ânus:

Epidermóide

Doença de Bowen

Sarcoma de Kaposi

- Câncer de canal anal: Epidermóide

Basalóide

Sarcoma

Alguns trabalhos relatam que vem ocorrendo aumento na incidência de câncer anal em homens mais jovens, principalmente em homossexuais e bissexuais ${ }^{2,13}$. Além disso, vários trabalhos correlacionam as doenças sexualmente transmissíveis com o risco de câncer anal ${ }^{9,13,14}$. Os dados da literatu-
- $\quad$ Perda de peso

- $\quad$ Alteração do H. I.

- Outros:
Carcinoma de células basais

Tumor de Buschke-Löwenstein

Doença de Paget

Epidermóide mucóide

Melanoma maligno

Adenocarcinoma

ra e da nossa pesquisa poderiam explicar o aumento de casos em homens mais jovens, que poderiam ter atividade sexual ano-receptiva.

A média de idade dos pacientes em nosso estudo foi 63,8 anos (24-91), com as mulheres apresentando uma média (66 anos) significativamente maior 
que os homens (59,4anos). Esses dados são similares aos encontrados na maioria dos trabalhos levanta$\operatorname{dos}^{4,6,12}$. Houve um predomínio do gênero feminino $(76,6 \%)$ e uma relação de 3,3 mulheres para cada homem, maior que o encontrado no trabalho de Nakajima et al., que foi de $2,4^{6}$ e menor que o valor encontrado no trabalho de Guimarães et al. ${ }^{11}$. O pico de incidência de câncer da região anal é entre 58-64 anos, mas nas últimas décadas vem ocorrendo aumento apreciável na incidência em homens mais jovens ${ }^{2}$. Em nossa pesquisa a faixa etária que mais concentrou pacientes com câncer anal foi entre 61 e 80 anos. Nakajima et al., analisando 58 pacientes relataram uma maior frequiência dos casos entre 60 e 90 anos $^{6}$.

O CEC foi o tipo histológico mais encontrado com 68\% (62) dos casos, um valor menor que o encontrado na literatura ${ }^{2,7,6}$, porém esse dado pode não corresponder a realidade, pois encontramos $8(10,2 \%)$ pacientes com adenocarcinoma de canal anal. Sabendo que o adenocarcinoma de reto é mais freqüente que o de canal anal ${ }^{1}$, esses casos poderiam ser uma extensão do adenocarcinoma de reto para o canal anal. Excluindo-se os adenocarcinomas da casuística, o CEC passaria a responder por $75 \%$ dos casos, em conformidade com os dados encontrados na literatura ${ }^{2,6,7}$.

Um tema de destaque, atualmente, é o papel do HPV como fator de risco para câncer anal. Em um estudo caso-controle, Frisch et al. identificaram o HPV em $88 \%$ dos 388 pacientes portadores de câncer de canal anal ${ }^{11}$. Vários estudos têm notado um aumento da incidência de neoplasia anal, principalmente em pessoas jovens e em áreas urbanas, sugerindo uma associação entre doenças sexualmente transmissíveis e câncer anal. Não foi o objetivo da nossa pesquisa estabelecer a relação entre as doenças sexualmente transmissíveis (DST) e o câncer de canal anal e ânus. Com a finalização do presente estudo demográfico, estaremos colocando em prática um protocolo mais completo, para que futuramente, possamos responder a essa e outras questões relacionadas ao câncer de canal anal e ânus.

\section{CONCLUSÃO}

Foram diagnosticados 91 casos de câncer anal em 13 anos de estudo. A incidência variou entre 0,18 e 0,83 por 100.000 pessoas, surgindo, em média, 0,39 novos casos de câncer por 100.000 pessoas a cada ano. A sétima e a oitava décadas de vida concentraram o maior número de casos. A maioria dos casos ocorreu entre as mulheres, sendo que o carcinoma epidermóide representou o tipo histológico mais prevalente. Em relação ao grau de diferenciação, os cânceres apresentavam moderada diferenciação na maior parte dos laudos de anátomo-patologia.

ABSTRACT: The lack of specific demographic studies about anal canal and anus cancer in Brazil and a better understanding of pathologies' behavior in Sergipe's state had motivated the accomplishment of such study. In retrospective analysis (1993 - 2005) we evaluate the main demographics characteristics of the anal cancer in Sergipe. From a total of 91 patients, $69(76.6 \%)$ were female and $21(23.4 \%)$ were male. The average of new cases a year was of 0,39 per 100.000 people, with standard deviation of $\pm 0,16$. The squamous cell carcinoma (SCC) account of $68 \%$ of the cases of anal cancer. In the present study it was observed an unconnected medical charts fulfillment and the necessity of a protocol for the assistance as well as the follow-up of the patients with anal canal and anus cancer.

Key words: Cancer, Anal cancer, Demographic study.

\section{REFERÊNCIAS}

1. CRUZ, G. M. G. Tumores Malignos no Ânus e Canal Anal. In: CRUZ, G. M. G. Coloproctologia - Propedêutica Nosológica. Rio de Janeiro: Revinter, 2000, p. 1187 - 1196.

2. LICITRA, L.; SPINAZZE, S.; DOCI, R.; EVANS, T. R. J.; TANUM, G.; DUCREUX, M. Cancer of the anal region. Critical Reviews in Oncology/Hematology. v. 43, p. 77 - 92, July, 2002.
3. Estimativa 2006 - Estimativa da Incidência de Câncer no Brasil. Instituto Nacional do Câncer - INCA, 21/08/2006. <http:/ /www.inca.gov.br/estimativa/2006/versaofinal.pdf>

4. MAGGARD, M. A.; BEANES, M. D.; Ko C. Y. Anal canal cancer: a population-based reappraisal. Disease Colon Rectum, v. 46, p. 1517 - 1524, November, 2003.

5. CRESS, R. D.; HOLLY, E. A. Incidence of anal cancer in California: increased incidence among men in San Francisco, 1973 - 1999. Preventive Medicine. v. 36, p. 555 - 560, 2003. 
6. NAKAJIMA, G. S.; SANTOS, R. T.; ALVES, V. A. F.; HABRGAMA, A. Carcinoma de ânus: aspectos epidemiológicos, histológicos, imuno-histológicos e por hibridação in situ. Arquivos Brasileiros de Cirurgia Digestiva. v. 14, p. 133 - 138, Julho/Setembro, 2001.

7. ESIASHVILI, N.; JEROME, L.; MATTHEWS, R. H. Carcinoma of the Anus: Strategies in Management. The Oncologist. v. 7, p. 188 - 199, March, 2002.

8. ROELOFSEN, F.; BARTELINK, H. Combined Modality Treatment of Anal Carcinoma. The Oncologist, v. 3, p. $413-$ 418, September, 1998.

9. População Residente em Sergipe. Censos Demográficos e Contagem populacional; para os anos intercensitários, estimativas preliminares dos totais populacionais, estratificados por idade e sexo pelo MS/SE/Datasus - 21/08/2006 <http:// tabnet.datasus.gov.br/cgi/tabcgi.exe?ibge/cnv/popse.htm >

10. FRISCH, M. On the etiology of anal squamous carcinoma. Danish Medical Bulletin. v. 49, n. 3, p. 194 - 208, May, 2002.

11. GUimARÃES, A. P.; MATOS, D.; SEGRETO, R.; FORONES, N. M. Carcinoma Espinocelular de Canal Anal: análise de 11 casos. Arq. Gastroenterologia, v. 38, n. 1, p. 9 13, Janeiro/Março, Julho/Setembro, 2001.
12. LARANGEIRA L. L. S.; ANDRADE S. K. V. Incidência de Carcinoma de Canal Anal na Regional de Saúde de Londrina (PR). Revista brasileira de Coloproctologia, v. 24, n. 3, p. 240 - 246, 2004.

13. FRISCH, M; GLIMELIUS, B.; VAN DE BRULE, A.; WOHLFAHRT, J.; MEIJER, J. L. M.; WALBOOMERS, J. M. M. et al. Sexually Transmitted Infection as a Cause of Anal Cancer. The New England Journal of Medicine, v. 337, n. 19, p. 1350 - 1358, November, 1997.

14. GOLDIE, S. J. The Clinical Effectiveness and Costeffectiveness of Screening for Anal Squamous Intraepithelial Lesions in Homosexual and Bisexual HIV-Positive Men. JAMA. v. 281, n. 19, p. 1822 - 1829, May, 1999.

15. WONG, S; GIBBS, P.; CHAO, M.; JONES, I.; MCLAUGHLIN, S.; TJANDRA, J. et al. Carcinoma of the anal canal: a local experience and review of the literature. ANZ. J. Surg, v. 74, p. 541 - 546, October, 2003.

\section{Endereço para Correspondência:}

JUVENAL DA ROCHA TORRES NETO

Rua Ananias Azevedo, 100, Ap. 902, Bairro: 13 de julho. 49020-080 - Aracaju (SE).

E-mail: Jtorres@infonet.com.br 